\title{
Biomimicry: an approach from the CumInCAD database
}

\author{
Frederico Braida \\ Universidade Federal de Juiz de Fora | Brasil | frederico.braida@ufff.edu.br \\ Mariana Alves Zancaneli \\ Universidade Federal de Juiz de Fora | Brasil | zancaneli.m@gmail.com \\ Isabela Gouvêa \\ Universidade Federal de Juiz de Fora | Brasil | isabela.gouvea@arquitetura.ufjf.br \\ Icaro Chagas \\ Universidade Federal de Juiz de Fora | Brasil | icaro.chagas@arquitetura.ufjf.br
}

\begin{abstract}
This article addresses the theme of biomimetics in the context of the interaction between architecture, urbanism, design and digital technologies. The main aim is to present the state of the art of the research published in the SIGraDI congresses and congresses of the sister associations. The methodology adopted was systematic literature and bibliometric review. The Com InCAD database was chosen as the data collection source. In the end, the text reveals in which associations the theme of biomimetics is more explored, as well as the authors who are most influential in this field of knowledge.
\end{abstract}

Keywords: Architecture; Nature; Biomimetics; Literature Review; CumlnCAD.

\section{INTRODUÇÃO}

O XXIV Congresso da Sociedade Ibero-americana de Gráfica Digital (SIGraDi), edição de 2020, propõe-se a refletir sobre o "Design transformador" e, para tanto, discute-se o papel crucial "das diferentes disciplinas projetuais para a manutenção da vida, por meio de uma sinergia com tecnologias convencionais e disruptivas", bem como as "convergências entre sistemas naturais e artificiais" (Jaramillo \& Torreblanca-Díaz, 2019). No âmbito dessa discussão, dentre outras temáticas, mostra-se relevante a abordagem sobre a "biomimética".

Portanto, cabe destacar que, dentre os nove eixos temáticos do SIGraDi 2020, tem-se o eixo 8, intitulado "Projeto biomimético e bioinspirado", o qual é composto pelos seguintes subtemas: "Design e arquitetura baseados na natureza", "Morfologias bioinspiradas", "Estruturas e tecnologias bioinspiradas" e "Metodologias e estratégias baseadas na natureza" (Sigradi, 2019).

O termo biomimética se origina da junção do termo "bios", que significa "vida", com o termo "mimesis", que significa "imitação" (Queiroz, Rattes \& Barbosa, 2017). Trata-se, pois, de uma noção de imitação de formas, conteúdos e funções biológicas ou naturais, a qual já tem sido empregada nos campos da arquitetura, urbanismo, artes e design há muito tempo (Benyus, 2002; Gruber, 2011). De acordo com Couceiro (2016, p. 36), "os biomorfismos presentes nos elementos ornamentais da arquitetura egípcia, grega e romana são provavelmente os elementos de caráter biomimético mais reconhecíveis dentro das referências arquitetônicas históricas". Mas também encontramos, na arquitetura islâmica, principalmente em elementos não figurativos, padrões fractais, inspirados na natureza. Esses padrões fractais estão presentes, também, por exemplo, na arte e na estruturação urbana do período pré-colonial da África Subsaariana. Assim, conforme destaca Couceiro (2016, p. 36), "a biomímesis é um processo intelectual que, ao largo da história, vem expressando, de forma intencional ou intuitiva, na diversas vertentes da arquitetura e do urbanismo".

Ainda segundo Couceiro (2016, p. 36), "a arquitetura biomimética é a arquitetura resultante de uma metodologia de investigação baseada na observação e na replicação de morfologias, mecanismos, processos e comportamentos provenientes de campos de estudo como a biologia, a biofísica, a biomecâmica, as neurociências, as matemáticas complexas e a cibernética". E é justamente a partir dessa perspectiva lógico-matemático-computacional que diversos processos criativos têm se dado levando-se em conta os hibridismos entre a informática e a biomimética. É sob esse prisma que a biomimética tem estado em pauta das discussões levadas a cabo nos congressos da SIGraDi e das associações irmãs.

De acordo com Knippers, Speck \& Nickel (2016, p. 1), "os recentes avanços na imaginação computacional, simulação e fabricação abriram uma nova era na biomimética: a diferenciação local em várias escalas, a principal característica das construções naturais que permitem vários princípios de trabalho em rede, pode pela primeira vez não apenas ser analisada, mas até certo ponto também ser transferido para a escala da construção civil". Portanto, nota-se que, com o desenvolvimento e disseminação das tecnologias CAD, CAAD e CAE, têm sido encontradas novas possibilidades de aplicação do biomimimetismo na arquitetura, no urbanismo e no design. 

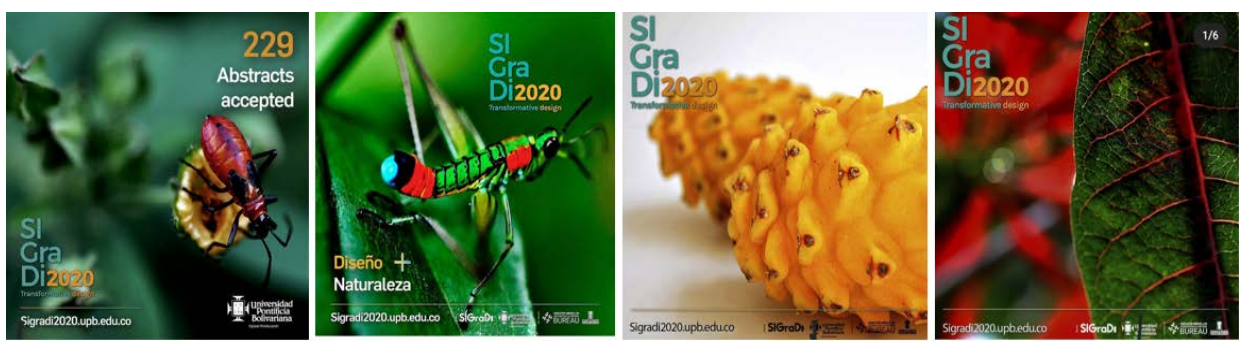

Figura 1: Série de imagens veiculadas no site e nas redes sociais do SIGraDi 2020. Fonte: https://www.instagram.com/sigradi_official/.

Tal como mencionam Braida et al. (2016, pp. 19-20), "uma nova função atribuída atualmente aos arquitetos é a de desenvolver ferramentas computacionais de criação, conhecidas como sistemas generativos de projeto, que se enquadram no conceito de arquitetura digital evolucionária e propõem o modelo de evolução da natureza como meio de obter soluções de projeto a partir da expressão de conceitos arquitetônicos e modelos de simulação e técnicas de otimização do desempenho de um edifício, seja, por exemplo, otimização da forma ou da estrutura. Neste contexto, a utilização de algoritmo genético tem sido frequente como métodos adaptativos de pesquisa heurística de otimização, simulando processos de evolução biológica na busca por melhores soluções para um determinado problema. A computação, enquanto busca de uma solução para um problema a partir de inputs (entradas) e outputs (resultados/saídas), possibilitou a proposição e viabilização de ambientes responsivos, nos quais as condições do espaço podem mudar e se adaptar a critérios predefinidos ou desejáveis (...)".

Essa discussão sobre a conciliação entre a tecnologia e a vida mostra-se plenamente pertinente, sobretudo para os dias de hoje, uma vez que há uma urgente demanda por soluções sustentáveis, que respeitem, lato sensu, o ecossistema. Ressalta-se que o design, a arquitetura e o urbanismo, entre outras disciplinas, têm buscado algumas respostas dentro desses paradigmas. No âmbito específico da abordagem da biomimética na era da cultura digital, verificam-se diversas contribuições significativas, dentre as quais podem ser mencionados as dos autores encontrados na revisão sistemática apresentada neste artigo.

Diante desse cenário e visando contribuir para a evidenciação do estado da arte e com a revisão das discussões acerca da biomimética já levadas a cabo no âmbito dos congressos da SIGraDi e das demais sociedades correlacionadas (ACADIA, CAADRIA, eCAADe, ASCAAD e CAAD futures), partiu-se da seguinte questão: Como a biomimética tem sido explorada pelos pesquisadores vinculados à SIGraDi e às sociedades irmãs?

Para responder a essa pergunta, foi realizada uma revisão sistemática de literatura a partir da base de dados CumlnCAD, a qual reúne os trabalhos publicados nos eventos científicos supracitados. Optou-se, metodologicamente, pela metanálise, a fim de integrar os resultados encontrados, conforme descrevem Costa \& Zoltowiski (2014), a partir de diversos outros autores. Tratase, portanto, de um artigo que se mostra predominantemente como um artigo de vertente bibliométrica (Chueke \& Amatucci, 2015).
Destaca-se, portanto, que a principal motivação para a pesquisa foi compreender como essa temática já vem sendo abordada ao longo dos anos pelos autores que têm participado dos eventos da SIGraDi e das demais sociedades, uma vez que, neste ano, em 2020, o tema principal do evento é o Design Transformador (Transformative Design), com um forte apelo para os temas relacionados à biomimética e à bioinspiração, conforme pode ser verificado nas imagens veiculadas no site e nas redes sociais do evento (Figura 1).

De fato, conforme propõe a abordagem do SIGraDi 2020, tem-se percebido a relevância da adoção da natureza como fonte de inspiração para diversos projetos, sobretudo quando se busca por soluções sustentáveis. As junções entre as tecnologias da natureza e as tecnologias digitais têm evidenciado um enorme potencial criativo na contemporaneidade.

Portanto, o principal objetivo deste artigo é apresentar uma revisão sobre como a biomimética, no âmbito da cultura digital, vem sendo enfocada por diferentes pesquisadores ao redor do mundo, a partir de um olhar para os dados encontrados na base CumlnCAD.

\section{METODOLOGIA}

Do ponto de vista metodológico, pode-se dizer que este artigo é fruto de uma revisão sistemática de literatura, com abordagem predominantemente bibliométrica, de metanálise (Mattos, 2004; Chueke \& Amatucci, 2015; Costa \& Zoltowiski, 2014). Cabe destacar que, de acordo com Chueke \& Amatucci $(2015$, p. 2), "particularmente, no campo das ciências sociais aplicadas os estudos bibliométricos se concentram em examinar a produção de artigos em um determinado campo de saber, mapear as comunidades acadêmicas e identificar as redes de pesquisadores e suas motivações". É nesse sentido que este artigo pretende contribuir.

Cabe mencionar que este artigo encontra inspiração metodológica nos artigos de revisão sistemática de literatura e de bibliometria, de Güzelci, Alaçam \& Güzelci (2019), de Sperling et al. (2019) e de Herrera e Braida (2019), publicados no eCAADe + SIGraDi 2019, os quais também tinham por objetivo fornecer subsídios para a compreensão do estado da arte, propor uma revisão histórica e servir de alicerce para futuras pesquisa.

Portanto, buscou-se, diante da coleção de artigos publicados na base de dados CumlnCAD, compreender como o biomimética tem sido abordada. $\mathrm{A}$ base de dados CumlnCAD <http://papers.cumincad.org/> foi selecionada 
como fonte para a coleta de dados, uma vez que se configura como "um índice cumulativo de publicações sobre projeto de arquitetura auxiliado por computador" e concentra publicações de pesquisadores de diferentes partes do mundo, incluindo "mais de 12.300 registros de periódicos e conferências como ACADIA, ASCAAD, CAADRIA, eCAADe, SiGraDi, CAAD Futures, DDSS e outros (CumlnCAD, 2020). Portanto, mostrou-se como a fonte adequada para a coleta dos dados. No entanto, cabe evidenciar, que, ao se escolher uma determinada base de dados, os resultados encontrados estão circunscritos a escopo da base, o que se apresenta como uma das limitações da pesquisa em tela.

Ainda, segundo Chueke \& Amatucci $(2015$, p. 2), partindo do pensamento de Okubo (1997), um dos objetivos da bibliometria é a "criação de indicadores que buscam sumarizar as instituições e os autores mais prolíferos, os acadêmicos mais citados e as redes de coautorias". Portanto, o que se buscou foi compreender em quais períodos, em quais eventos e a partir de quais autores a discussão sobre a biomimética esteve mais proeminente.

Levando-se em consideração os passos apresentados por Costa \& Zoltowiski (2014, p. 56), compilados de Akobeng (2005), a estruturação da pesquisa se deu em cinco etapas esquematizados na Figura 2, a saber:

(1) delimitação da questão a ser pesquisada (o problema da pesquisa);

(2) escolha das fontes de dados (a base de dados consultada);

(3) eleição das palavras-chave para a busca;

(4) busca e armazenamento dos dados; e

(5) tratamento, análise e apresentação dos dados encontrados.

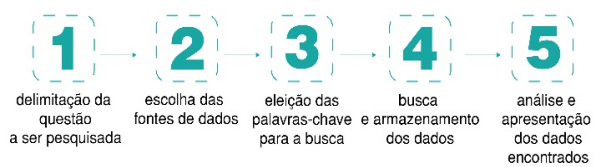

Figura 2: Percurso metodológico da pesquisa. Fonte: dos autores.

Na pesquisa, foram considerados os artigos levantados a partir de buscas com a palavra-chave "biomimética" e suas variações, em português, inglês e espanhol. Esses idiomas foram escolhidos por serem as línguas oficiais dos eventos da SIGraDi. Assim, foram contemplados os vocábulos:

(1) "biomimética";

(2) "biomimicry";

"(3) biomimetics";

(4) "biomimético";

(5) "biomimetic";

(6) "biomímese";
(7) "biomimesis";

(8) "biomímesis"; e

(9) "biomimetismo".

O Quadro 1 apresenta as palavras pesquisadas agrupadas por idioma.

Quadro 1: Idiomas e palavras pesquisadas. Fonte: dos autores.

\begin{tabular}{|c|c|}
\hline \multicolumn{2}{|c|}{ Língua das palavras pesquisadas } \\
\hline Português & $\begin{array}{c}\text { "biomimética"; "biomimético"; } \\
\text { "biomímese"; "biomimetismo". }\end{array}$ \\
\hline Espanhol & "biomímesis"; "biomimesis"; \\
\hline Inglês & "biomimicry"; "biomimetics"; \\
& "biomimetic"; \\
\hline
\end{tabular}

As buscas foram realizadas entre os dias 02 e 06 de março de 2020. Todos os dados encontrados foram registrados em uma planilha eletrônica composta pelos seguintes campos:

(1) Autoria;

(2) Ano de publicação;

(3) Título;

(4) Link de acesso ao artigo;

(5) Evento em que o artigo foi publicado; e

(6) Palavras-chave.

Dentro da planilha, foi criada uma página para cada palavra-chave pesquisada e encontrada, totalizando, portanto, cinco páginas. Além dessas, foi também acrescentada uma página na qual foram compilados todos os artigos encontrados, excluídas as coincidências (repetições)

Observa-se que as buscas foram realizadas manualmente, por meio do buscador da base de dados CumInCAD, não contando com a ajuda de nenhum software especializado para a coleta dos dados. Para minimizar as possibilidades de erros na quantificação dos dados coletados, foram realizadas duas etapas de verificação, garantindo a consistência, integridade e correção do levantamento.

A partir dessa planilha foram realizadas todas as análises, durante a última etapa da pesquisa. Deve-se ressaltar que foram adotados como critérios de análise: (a) ano das publicações, (b) autores, (c) principais subtemas discutidos, (d) origem das publicações; (e) palavras-chave dos artigos e (f) autores mais citados nas referências.

Assim, buscou-se levantar tanto os autores que mais publicaram sobre a temática, quanto os autores mais citados em todos os artigos, contribuindo para o 
mapeamento da comunidade que tem levado adiante pesquisas sobre a biomimética sob o ponto de vista da gráfica digital, da computação, da informática ou das tecnologias digitais nos campos da arquitetura, urbanismo e design.

\section{RESULTADOS E DISCUSSÃO}

Ao todo, foram encontrados, na base de dados CumInCAD, 78 artigos, que possuíam as seguintes palavras em qualquer parte do texto (Figura 3): "biomimética" (1), "biomimicry" (29), "biomimetics" (29), "biomimético" (0), "biomimetic" (35), "biomímese" (0), "biomimesis" (3), "biomímesis" (0) e "biomimetismo" (0).

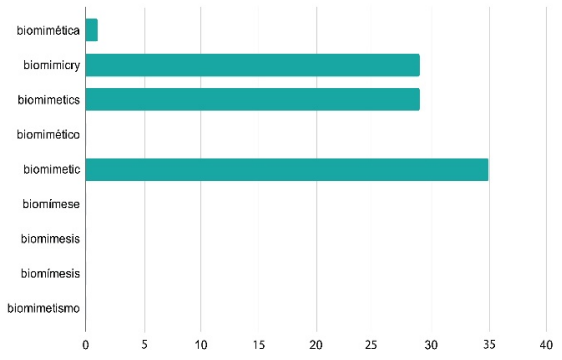

Figura 3: Recorrência das palavras nos artigos pesquisados. Fonte: dos autores.

Assim, destaca-se que apenas as palavras "biomimética", "biomimicry", "biomimetics", "biomimetic" e "biomimesis" foram encontradas, sendo que a maior recorrência foi de artigos que continham a palavra "biomimetic". As demais palavras pesquisadas não foram encontradas.

Quando analisadas as palavras presentes somente nos títulos das publicações, encontrou-se: "biomimética" (1), "biomimicry" (0), "biomimetics" (1), "biomimetic" (13) e "biomimesis" (0). Assim é relevante ponderar que o termo "biomimetic" tem sido o mais adotado nas publicações, especialmente nos títulos.

Sobre esses termos, Queiroz, Rattes \& Barbosa (2017, p. 126) relatam que eles começaram a aparecer na literatura a partir de 1950, referindo-se a "associações de atividades humanas frente à natureza". O termo biomimetics (biomimética) "foi usado pela primeira vez pelo engenheiro biomédico Otto H. Schmitt na década de 1950, objetivando criar uma distinção da Biofísica" (Queiroz, Rattes \& Barbosa, 2017, p. 126).

Já o termo biomimicry (que também pode ser traduzido por biomimética em língua portuguesa) é mais recente e data da década de 1970, sendo "criado pelo casal John Todd e Nancy Jack-Todd, a partir do grupo chamado The New Alchemy Institute" (Queiroz, Rattes \& Barbosa, 2017, p. 127). Os autores afirmam que "segundo o grupo, as necessidades humanas fundamentais poderiam encontrar soluções mais sustentáveis a partir do estudo da ecologia, biologia e da abordagem de sistemas bio-cibernéticos. Ou seja, observa-se a intenção de compreender a natureza e realizar uma associação harmoniosa entre mundo artificial - idealizada pelos homens - e mundo natural" (Queiroz, Rattes \& Barbosa, 2017, p. 127). É a esse universo que o termo biomimética se refere.
Ao se observar a distribuição dos artigos por ano, tem-se: 2005 (1), 2006 (1), 2007 (0), 2008 (9), 2009 (7), 2010 (2), 2011 (3), 2012 (8), 2013 (5), 2014 (9), 2015 (7), 2016 (6), 2017 (9), 2018 (4) e 2019 (7).

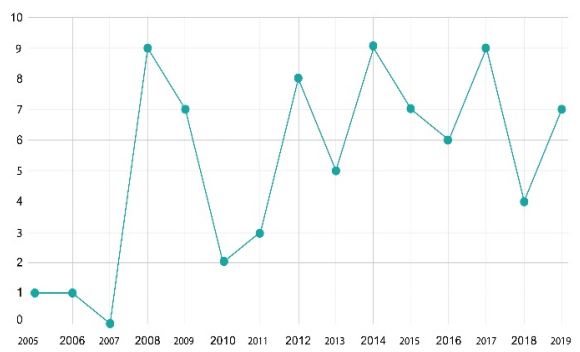

Figura 4: Distribuição dos artigos encontrados por ano. Fonte: dos autores.

Conforme pode ser percebido pelo gráfico da Figura 4, dentre as publicações analisadas ao longo dos tempos, os anos de 2008, 2014 e 2017 foram os que mais se destacaram, sendo publicados nove artigos em cada um desses anos, nos quais algumas das palavras-chave pesquisadas foram mencionadas.

Observa-se que, em 2008, o congresso a ACADIA abordou especificamente essa temática, tendo como tema principal "Silicon + skin: biological processes and computation" ("Silício + pele: processos biológicos e computação"). Nos demais anos em que houve maiores números de publicações sobre a temática, incluindo o ano de 2012, não foram encontrados eventos que apresentassem de forma clara o tema da biomimética, apenas alguns temas relacionados às tecnologias digitais em geral, como fabricação digital e design paramétrico.

É interessante destacar que o primeiro artigo dessa série histórica foi publicado no congresso da SIGraDi, em 2005, intitulado "New processes of digital design: intelligent skin" ("Novos processos de design digital: pele inteligente") e é de autoria de María Celeste Ibarrola, Vanesa Soledad lozzo e Sabrina Klor.

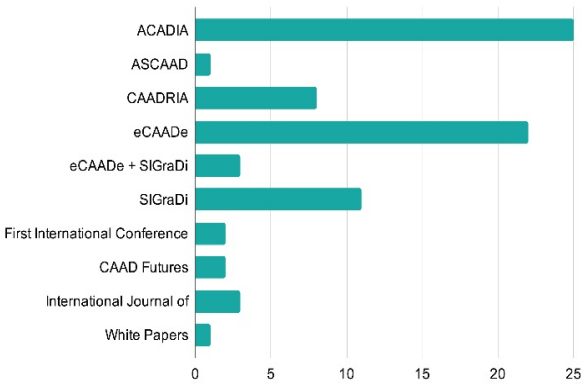

Figura 5: Distribuição dos artigos pesquisados segundo as fontes de publicação. Fonte: dos autores.

Quando se verifica a origem das publicações, constata-se: ACADIA (25), ASCAAD (1), CAADRIA (8), eCAADe (22), eCAADe + SIGraDi (3), SIGraDi (11); First International Conference on Critical Digital (2); CAAD Futures (2); 
International Journal of Architectural Computing (3); e White Papers (1). Logo, percebe-se, conforme Figura 5, que a temática da biomimética tem sido mais explorada no âmbito da ACADIA e do eCAADe.

Inclusive, se somados os artigos publicados pelo eCAADe com os artigos publicados pelo eCAADe + SIGraDi (frutos de um evento conjunto realizado em 2019, no Porto, em Portugal), o total de artigos se iguala aos publicados pela ACADIA, contabilizando 25 artigos para cada associação.

Acredita-se que essa temática ganhará maior repercussão no SIGraDI 2020, devido ao enfoque específico no "design transformador" e por contar com uma seção exclusiva para "Projeto biomimético e bioinspirado". Entretanto, deve-se ressaltar que os pesquisadores dessa área ainda representam um nicho bastante específico. Dos 328 resumos submetidos para o SIGraDi 2020, apenas 15 foram dirigidos para a seção "[08] Biomimética e projeto bioinspirado" (Jaramillo \& Días, 2020).

Porém, quando analisamos os autores que têm publicado sobre a temática ao longo do tempo, encontramos uma grande diversidade. Dos 78 artigos encontrados, 65 possuem autores diferenciados, o que sinaliza que tais autores não possuem uma tradição de investigação específica dentro dessa temática.

No entanto, cinco autores se destacam por terem publicados mais de um artigo sobre biomimética ao longo dos anos. Weston, Erdine e Krieg publicaram, entre 2010 e 2016, três artigos cada um. Sabin e Winn tiveram dois artigos cada, publicados entre 2012 e 2019 (Quadro 2).

Quadro 2: Recorrência dos autores e anos das publicações. Fonte: dos autores.

\begin{tabular}{|l|c|c|}
\hline \multicolumn{1}{|c|}{ Autor } & Total de artigos & Anos \\
\hline Weston & 3 & $2010 ; 2011 ; 2013$ \\
\hline Erdine & 3 & $2013 ; 2014 ; 2015$ \\
\hline Krieg & 3 & $2011 ; 2012 ; 2012$ \\
\hline Sabin & 2 & $2018 ; 2019$ \\
\hline Winn & 2 & $2012 ; 2015$ \\
\hline
\end{tabular}

Embora a temática de fundo desses 13 artigos seja a biomimética, esses autores apresentam abordagens bastante variadas, perpassando pelas questões da robótica, formais, de conforto ambiental etc.

O tema mais abordado dentre os artigos desses autores supracitados foi o design computacional e a fabricação robótica, presente nos seguintes artigos: "Performative architectural morphology: robotically manufactured biomimetic finger-joined plate structures" ("Morfologia arquitetônica performativa: estruturas de placas biomiméticas fabricadas por robô") (Krieg et al., 2011), "Computational design of robotically manufactured plate structures based on biomimetic design principles derived from clypeasteroida" ("Projeto computacional de estruturas de placas fabricadas roboticamente com base em princípios de projeto biomimético derivados de clypeasteroida") (Krieg et al., 2012), "Re-framing architecture for emerging ecological and computational design trends for the built ecology" ("Reestruturando a arquitetura para tendências emergentes de design ecológico e computacional para a ecologia construída"),
(Winn, Vollen \& Dyson, 2012), "Transient thermal exchange and developmental form for tactile surfaces" ("Troca térmica transitória e forma de desenvolvimento para superfícies táteis") (Winn, 2015) e "Machinic morphospaces: biomimetic design strategies for the computational exploration of robot constraint spaces for wood fabrication" ("Morfoespaços maquínicos: estratégias de design biomimético para a exploração computacional de espaços de restrição de robôs para fabricação de madeira") (Schwinn et al., 2012), "Lumen" (Lúmen) (Sabin et al., 2018) e "Lumen" (Lúmen) (Sabin, 2019).

Já o tema do sombreamento solar foi debatido nos seguintes artigos: "Anisotropic Operations: A study in directed material weakening for solar shading" ("Operações anisotrópicas: um estudo sobre enfraquecimento direcionado de material para sombreamento solar") (Weston, 2011), "Memory Mesh: Conformationally Adaptive Solar Shading" ("Malha de memória: sombreamento solar adaptável") (Weston, 2010) e "Sweetgum Panels" ("Painéis de Sweetgum") (Weston \& Greenberg, 2013).

"Generative processes in tower design: simultaneous integration of tower subsystems through biomimetic analogies" ("Processos generativos no projeto de torres: integração simultânea de subsistemas de torres através de analogias biomiméticas") (Erdine, 2015) e "Biomimetic strategies in tower design" ("Estratégias biomiméticas no projeto de torres") (Erdine, 2013) discutem acerca da biomimética em projetos de torres. Por fim, o artigo "Reprogramming architecture - learning via practical methodologies" ("Reprogramando a arquitetura aprendizagem através de metodologias práticas") (Erdine \& Kallegias, 2014) se trata de um estudo das abordagens pedagógicas nas faculdades de arquitetura.

Tratando-se das palavras-chave declaradas nos artigos pesquisados, percebeu-se uma maior ocorrência, dentre as palavras-chave elencadas para a pesquisa, dos termos "biomimetics" (24) e "biomimicry" (23), sendo que todos os demais termos apareceram com frequência inferior a dez vezes.

Para a construção da nuvem de palavras da Figura 6, foram excluídos os termos que ocorreram somente uma vez.

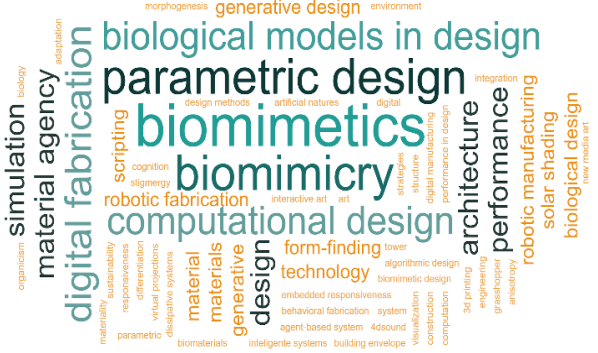

Figura 6: Principais palavras-chave encontradas nos artigos pesquisados.

Percebe-se que os artigos encontrados com as palavraschave pesquisadas se relacionam, recorrentemente, com os seguintes termos:

(1) "parametric design" (design paramétrico); 
(2) "computational design" (design computacional);

(3) "biological models in design" (modelos biológicos em design); e

(4) "digital fabrication" (fabricação digital).

Quando são analisadas as referências encontradas nos artigos, observamos uma recorrência de alguns autores que se destacam.

O Quadro 3 apresenta a lista de autores que apareceram, no mínimo, dez vezes na lista de referências bibliográficas, considerando todos os artigos e as diferentes formas de citação. Ao todo, são 11 autores que foram mais citados.

Quadro 3: Autores mais citados nas referências dos artigos analisados. Fonte: dos autores.

\begin{tabular}{|c|c|c|}
\hline \multicolumn{3}{|c|}{ Autores citados nas referências dos artigos analisados } \\
\hline Citado como & Quantidade de citaç̄es & Total de ciltacöes \\
\hline A. Menges & 7 & \multirow{4}{*}{52} \\
\hline Achim Menges & 4 & \\
\hline Menges, $\mathrm{A}$. & 33 & \\
\hline Menges, Achim & 8 & \\
\hline J. Knippers & 5 & \multirow{4}{*}{29} \\
\hline Jan Knippers & 4 & \\
\hline Knippers J & 19 & \\
\hline Knippers, Jan & 1 & \\
\hline Hensel, $\mathrm{M}$. & 20 & \multirow{2}{*}{23} \\
\hline Hensel, Michael Ulrich & 3 & \\
\hline Benyus, J. & 6 & \multirow{4}{*}{17} \\
\hline Benyus, J. M. & 9 & \\
\hline Benyus, Janine & 1 & \\
\hline Benyus, Janine M. & 1 & \\
\hline Oxman, $\mathrm{N}$ & 11 & \multirow{2}{*}{13} \\
\hline Oxman, Neri & 2 & \\
\hline Weinstock, M. & 8 & \multirow{4}{*}{13} \\
\hline Weinstock, Michael & 2 & \\
\hline M. Weinstock & 2 & \\
\hline Michacl Wcinstock & 1 & \\
\hline Speck, $\mathrm{T}$. & 9 & \multirow{3}{*}{12} \\
\hline T. Speck & 1 & \\
\hline Thomas Speck & 2 & \\
\hline Vincent, J. & 7 & \multirow{3}{*}{11} \\
\hline VIncent, JFV & 1 & \\
\hline Vincent, Julian & 3 & \\
\hline Kolarevic. B & 8 & \multirow{2}{*}{10} \\
\hline Kolarevic, Branko & 2 & \\
\hline Prusinkiewicz, $\mathrm{P}$. & 10 & 10 \\
\hline Schwinn, $T$ & 6 & \multirow{3}{*}{10} \\
\hline Schwinn, Tobias & 1 & \\
\hline Tobias Schwinn & 3 & \\
\hline
\end{tabular}

O autor mais citado é Achim Menges, um dos autores do livro "Emergent technologies and design: towards a biological paradigm for architecture" ("Tecnologias e design emergentes: rumo a um paradigma biológico para a arquitetura"), publicado em 2010, em coautoria com Michael Hensel e Michael Weinstock, que também aparecem na lista acima dentre os autores mais citados.

Vale também mencionar que Jan Knippers é o segundo autor mais citado. Ele é um dos coautores do livro
"Biomimetic research for architecture and building construction: biological design and integrative structures: biologically-inspired system" ("Pesquisa biomimética para arquitetura e construção civil: projeto biológico e estruturas integrativas: sistema de inspiração biológica"), publicado em 2016.

Pode-se verificar que esses autores presentes no Quadro 3 constituem uma lista de referências para a temática da biomimética aplicada no contexto da arquitetura, urbanismo, artes, design e engenharia, em interface com os campos da computação, informática e das tecnologias digitais. Além dessas áreas, os artigos pesquisados revelam, assim como destacado por Knippers, Speck \& Nickel (2016), que a abordagem da biomimética é transversal e interdisciplinar, envolvendo, também, disciplinas como a matemática e a biologia.

\section{CONSIDERAÇÕES FINAIS}

Conforme buscamos evidenciar neste artigo, o tema da biomimética já vem sendo abordado nos congressos da SIGraDi e das socieadades irmãs desde 2005, como podese constatar ao se fazer uma busca pelos artigos cadastrados na base de dados CumInCAD.

Os temas enfocados são bastante diversos, não sendo possível constituir um núcleo sólido de abordagem, ainda que os artigos possuam como palavras-chave mais recorrentes "parametric design", "computational design", "biological models in design" e "digital fabrication".

Embora os autores também sejam diversificados, fo possível encontrar tanto autores que já publicaram mais de um artigo sobre essa temática, bem como autores que são amplamente citados nos diversos artigos. Esses autores constituem uma lista de referências sobre a temática e revelam parte do lastro teórico a partir do qual os pesquisadores principalmente dos campos da arquitetura, urbanismo e design têm conduzido suas investigações quando se trata dos projetos inspirados na natureza.

Pode-se dizer, em consonância com os autores que explicitam os benefícios das pesquisas bibliométricas, que a principal contribuição deste artigo é apresentar uma revisão sistematizada das publicações que versam sobre as pesquisas que têm por escopo o tema do projeto bioinspirado, à luz das tecnologias digitais.

Deve-se ainda recuperar que a revisão apresentada neste artigo se propõe a servir de base para outras pesquisas sobre o tema e para contribuir para o estabelecimento do estado da arte sobre a biomimética no âmbito das sociedades que integram a base de dados CumInCAD, tendo por motivação o principal escopo de discussão proposta para o SIGraDi 2020: o design transformador.

Por fim, sugere-se, como desdobramento futuro deste artigo, a incorporação dos artigos relacionados ao tema publicados no SIGraDi 2020. Assim como já mencionado, acredita-se que o SIGraDi 2020 oferecerá uma importante contribuição para o avanço das abordagens sobre essa temática, uma vez que, embora a biomimética já venha sendo discutida desde 2005 , neste ano, os processos entre os sistemas naturais e os artificiais supostamente fomentarão uma ampla reflexão sobre a biomimética como saber transversal e interdisciplinar. 


\section{CONSIDERAÇÕES FINAIS}

Os autores agradecem à Pró-Reitoria Pós-Graduação e Pesquisa da Universidade Federal de Juiz de Fora (PROPP/UFJF), ao Conselho Nacional de Desenvolvimento Científico e Tecnológico (CNPq), à Coordenação de Aperfeiçoamento de Pessoal de Nível Superior (Capes) e à Fundação de Amparo à Pesquisa do Estado de Minas Gerais (Fapemig) pelas concessões das bolsas de iniciação científica e de mestrado.

Os autores também agradecem a todos os membros do Laboratório de Estudos das Linguagens e Expressões da Arquitetura, Urbanismo e Design (LEAUD), um grupo de pesquisa cadastrado no Diretório de Grupos do CNPq, vinculado à Faculdade de Arquitetura e Urbanismo da UFJF e ao Programa de Pós-Graduação em Ambiente Construído da UFFJF.

\section{REFERÊNCIAS}

Akobeng A. K. (2005). Understanding systematic reviews and meta-analysis. Archives of disease in childhood, 90(8), 845848. Retried on $6^{\text {th }}$ April, 2020 on https://doi.org/10.1136/adc.2004.058230.

Benyus, J. M. (2002) Biomimicry: innovation inspired by nature. New York: William Morrow.

Braida, F., Lima, F., Fonseca, j. \& Morais, V. (Orgs.). (2016). 101 conceitos de arquitetura e urbanismo na era digital. São Paulo: ProBooks.

Chueke, G. V., \& Amatucci, M. (2015). O que bibliometria? Uma introdução ao Fórum. Revista Eletrônica de Negócios Internacionais, 10 (2), 1-5. Recuperado em 6 de abril, 2020 de http://dx.doi.org/10.18568/1980-4865.1021-5

Costa, A. B. \& Zoltowiski, A. P. C. (2014). Como escrever um artigo de revisão sistemática. In Koller, S. H., Couto, M. C. P. P. \& Hohendorff, J. V. (Orgs.). Porto Algere: Penso. p.55-70.

Couceiro, M. C. (2016). Biomimética. In Braida, F., Lima, F., Fonseca, j. \& Morais, V. (Orgs.). 101 conceitos de arquitetura e urbanismo na era digital. São Paulo: ProBooks. p.36-37.

CumlnCAD (2020). About. Cumulative Index about publications in Computer Aided Architectural Design. Recuperado em 9 de junho, 2020 de http://papers.cumincad.org/about.html.

Erdine, E. (2013). Biomimetic Strategies in Tower Design. Proceedings of the 31 st eCAADe Conference. Recuperado em 20 julho, 2020 de http://papers.cumincad.org/cgibin/works/paper/ecaade2013_192

Erdine, E. (2015). Generative Processes in Tower Design: Simultaneous Integration of Tower Subsystems Through Biomimetic Analogies. Proceedings of the 35th Annual Conference of the Association for Computer Aided Design in Architecture (ACADIA). Recuperado em 20 julho, 2020 de http://papers.cumincad.org/cgi-bin/works/paper/acadia15_173

Erdine, E., \& Kallegias, A. (2014). Reprogramming Architecture Learning via Practical Methodologies. Proceedings of the 32 nd eCAADe Conference. Recuperado em 20 julho, 2020 de http://papers.cumincad.org/cgibin/works/Show?ecaade2014_137.

Gruber, P. (2011). Biomimetics in architecture: architecture of life and buildings. New York: SpringerWienNewYork.

Güzelci, O. Z., Alaçam, S. \& Güzelci, H. (2019). "Trend Topics and Changing Concepts of Computational Design in the Last 16 Years A content analysis", p. 423-430. In Proceedings of 37 eCAADe and XXIII SIGraDi Joint Conference, "Architecture in the Age of the 4Th Industrial Revolution", Porto 2019, Sousa, José Pedro; Henriques, Gonçalo Castro; Xavier, João Pedro (eds.). São Paulo: Blucher.
Herrera, P. \& Braida, F. (2019). "Digital Technologies in Latin American Architecture A Literature Review from the Third to the Fourth Industrial Revolution", p. 431-442. In Proceedings of 37 eCAADe and XXIII SIGraDi Joint Conference, "Architecture in the Age of the 4Th Industrial Revolution", Porto 2019, Sousa, José Pedro; Henriques, Gonçalo Castro; Xavier, João Pedro (eds.). São Paulo: Blucher, 2019.

Jaramillo, J. R; Torreblanca-Díaz, David A. (2019) Convite.SIGraDi 2020. Recuperado em 9 junho, 2020 de https://sigradi2020.upb.edu.co/pt/congresso/.

Jaramillo, J. R; Torreblanca-Díaz, David A. (2020). Asignación de resúmenes para revisores / designação de revisores para os resumos / SIGraDi 2020. E-mail. Recebido em 8 abril, 2020.

Knippers, J.; Speck, T.; Nickel, K. G. (2016). Biomimetic research: a dialogue between the disciplines. In: Biomimetic research for architecture and building construction: biological design and integrative structures: biologically-inspired system. Cham: Springer. pp. 1- 5 .

Krieg, O. D.; Dierichs, K.; Reichert, S.; Schwinn, T.; Menges, A. (2011). Performative Architectural Morphology: Robotically manufactured biomimetic finger-joined plate structures. Proceedings of the 29th eCAADe Conference. Recuperado em 20 julho, 2020 de http://papers.cumincad.org/cgibin/works/paper/ecaade2011_035.

Krieg, O. D.; Mihaylov, B.; Schwinn, T.; Reichert, S:; Menges, A (2012). Computational Design of Robotically Manufactured Plate Structures Based on Biomimetic Design Principles Derived from Clypeasteroida. Proceedings of the 30th eCAADe Conference. Recuperado em 20 julho, 2020 de http://papers.cumincad.org/cgibin/works/paper/ecaade2012_152

Mattos, P. L. C. L. de. (2004) Bibliometria: a metodologia acadêmica convencional em questão. RAE-eletrônica, 3 (2), 1 6. Recuperado em 6 abril, 2020 de http://www.rae.com.br/eletronica/index.cfm?FuseAction=Artig o\&ID=2199\&Secao=DEBATE\&Volum $\mathrm{e}=3 \&$ Numero $=2 \&$ Ano $=2004$.

Okubo, Y. (1997). bibliometric indicators and analysis of research systems: methods and examples. OECD Science, Technology and Industry Working Papers, OECD Publishing. Recuperado em $15 \quad$ junho, 2020 de http://dx.doi.org/10.1787/208277770603.

Queiroz, N., Rattes, R. \& Barbosa, R. (2017). Biônica e Biomimética no contexto da complexidade e sustentabilidade em projeto. p. 127 -144. In Design \& Complexidade. São Paulo: Blucher.

Sabin, J. (2019). Lumen. White papers do Living Architecture Systems Group 2019, Riverside Architectural Press: Toronto, Canadá 2019. pp.291 - 318. Recuperado em 20 julho, 2020 de http://papers.cumincad.org/cgibin/works/paper/lasg_whitepapers_2019_291.

Sabin, J.; Pranger, D.; Binkley, C.; Strobel, K.; Liu, J. (2018). Lumen. Proceedings of the 38th Annual Conference of the Association for Computer Aided Design in Architecture (ACADIA). Recuperado em 20 julho, 2020 de http://papers.cumincad.org/cgibin/works/paper/acadia18_444.

Sigradi (2019). Chamadas. SIGraDi 2020. Recuperado em 9 junho, 2020 de https://sigradi2020.upb.edu.co/pt/congresso/.

Schwinn, T.; Krieg, O.D.; Menges, A.; Mihaylov, B.; Reichert, S. (2012). Machinic Morphospaces: Biomimetic Design Strategies for the Computational Exploration of Robot Constraint Spaces for Wood Fabrication. Proceedings of the 32nd Annual Conference of the Association for Computer Aided Design in Architecture (ACADIA). Recuperado em 20 julho, 2020 de http://papers.cumincad.org/cgibin/works/paper/acadia12_157.

Sperling, D.; Vizioli, S. H. T., Botasso, G. B., Tiberti, M. S., Santana, E. F. Z., Sígolo \& B. O. O. (2019). "Crossing Timelines Main research topics in the histories of eCAADe and 
SIGraDi", p. 407-416 . In Proceedings of 37 eCAADe and XXIII SIGraDi Joint Conference, "Architecture in the Age of the 4Th Industrial Revolution", Porto 2019, Sousa, José Pedro; Henriques, Gonçalo Castro; Xavier, João Pedro (eds.). São Paulo: Blucher, 2019.

Weston, M. (2010). Memory Mesh: Conformationally Adaptive Solar Shading. Proceedings of the 14th Congress of the Iberoamerican Society of Digital Graphics. Recuperado em 20 julho, 2020 de http://papers.cumincad.org/cgibin/works/Show?sigradi2010_412.

Weston, M. (2011). Anisotropic Operations: A study in directed material weakening for solar shading. 29th eCAADe Conference Proceedings. Recuperado em 20 julho, 2020 de http://papers.cumincad.org/cgibin/works/Show?ecaade2011_161
Weston, M., \& Greenberg, D. (2013). Sweetgum Panels. Proceedings of the 31st eCAADe Conference. Recuperado em 20 julho, 2020 de http://papers.cumincad.org/cgibin/works/Show?acadia09_216.

Winn, K.; Vollen, J.; Dyson, A. (2012). Re-Framing Architecture for Emerging Ecological and Computational Design Trends for the Built Ecology. Recuperado em 20 julho, 2020 de http://papers.cumincad.org/cgibin/works/Show?acadia12_251.

Winn; K. (2015). Transient Thermal Exchange and Developmental Form for Tactile Surfaces. Proceedings of the 35th Annual Conference of the Association for Computer Aided Design in Architecture (ACADIA). Recuperado em 20 julho, 2020 de http://papers.cumincad.org/cgibin/works/paper/ecaade2015_230. 